\title{
Multiarm Aniline Oligomers: Molecular Architecture, Self-Assembly, and Electrochromic Performance
}

Meng Wang, Jie Fan, Hao Lu, Mingming Huang, Xiaoyao Qiao, Jiping Yang*

Key Laboratory of Aerospace Advanced Materials and Performance, Ministry of Education, School of Materials Science and Engineering, Beihang University, Beijing 100191, China

\section{EXPERIMENTAL SECTION}

\section{Materials}

N-phenyl-1,4-phenylenediamine (Alfa Aesar), 4,4'-diaminodiphenylamine sulfate hydrate (TCI), succinic anhydride (Sinopharm Chemical Reagent Co., Ltd, China), terephthalic acid (innoche), isophthalic acid (Adamas), 1,3,5-benzenetricarboxylic acid (Sinopharm Chemical Reagent Co., Ltd, China), 2-Acrylamido-2-methylpropane sulfonic acid (AMPS, TCI) and ammonium persulphate (APS, Beijing Chemical Works, China) were used as received without further purification. N, N'dimethylformamide (DMF) and N, N'-dimethyl acetamide (DMAc) were used after dehydration. All other chemicals were purchased from Beijing Chemical Works, China and used as received. FTO conducting glass ( $\leqslant 15 \Omega /$ sq) was purchased from Zhuhai Kaivo Optoelectronic Technology Co., Ltd. 


\section{Synthesis of tetraaniline derivatives}

NH2-(Ani)4-COOH: At room temperature, N-phenyl-1,4-phenylenediamine was reacted with equimolar succinic anhydride to get succinic acid-protected aniline dimer. Then, the succinic acidprotected aniline dimer $(1.43 \mathrm{~g}, 5.0 \mathrm{mmol})$ and 4,4'-diaminodiphenylamine sulfate hydrate $(1.49 \mathrm{~g}$, $5.0 \mathrm{mmol})$ were dissolved into the mixed solution of DMF $(100 \mathrm{ml})$, distilled water $(15 \mathrm{ml})$, and hydrochloric acid $(15 \mathrm{ml})$. The ammonium persulfate solution (1.14 $\mathrm{g}$ APS dissolved in $50 \mathrm{ml}$ of 1.0 $\mathrm{mol} / \mathrm{L} \mathrm{HCl}$ ) was dropwise added to the mixture. After being stirred constantly at $-5{ }^{\circ} \mathrm{C}$ for $1 \mathrm{~h}$, the mixture was poured to the distilled water and filtered. Then the coarse product was reduced by hydrazine hydrate. Soxhlet extraction was used to remove the by-products with 1,2-dichloroethane and THF. After vacuum drying, the leucoemeraldine base (LEB) $\mathrm{NH}_{2}-(\text { Ani })_{4}-\mathrm{COOH}$ was obtained. Yield: 1.90 g, 65.0\% [1,2]. ${ }^{1} \mathrm{H}-\mathrm{NMR}\left(400 \mathrm{MHz}, \mathrm{DMSO}-\mathrm{d}_{6}\right), \delta$, ppm: 9.71 (s, 1H, -CO-NH-), 8.00 (s, 2H, Ar-H), 7.47 (d, 2H, Ar-H), 7.19 (t, 3H, -NH-), 7.00-6.76 (m, 12H, Ar-H).

Bi(p)-(Ani)4-COOH: $0.831 \mathrm{~g}(5.0 \mathrm{mmol})$ of terephthalic acid, $20 \mathrm{ml}$ of thionyl chloride and a drop of DMF were added to a $50 \mathrm{ml}$ single-mouth flask. After refluxed at $85^{\circ} \mathrm{C}$ for $4 \mathrm{~h}$, the reaction solvent was removed by rotary evaporation to obtain terephthaloyl chloride. $0.962 \mathrm{~g}$ (2.0 mmol) LEB $\mathrm{NH}_{2}$-(Ani) ${ }_{4}-\mathrm{COOH}$ was dissolved in $30 \mathrm{ml}$ anhydrous $\mathrm{N}, \mathrm{N}^{\prime}$-dimethylacetamide (DMAc), protected with nitrogen, and pre-cooled to $0{ }^{\circ} \mathrm{C}$ in an ice bath. Then, $0.202 \mathrm{~g}(1.0 \mathrm{mmol})$ terephthaloyl chloride was dissolved in $10 \mathrm{ml}$ anhydrous DMAc and slowly added dropwise to the above solution. After that, the react was lasted for $36 \mathrm{~h}$ at room temperature. The reaction solution was poured into a large amount of deionized water, precipitated, and filtered under reduced pressure, then the precipitation was fully washed with deionized water, acetone and isopropanol. The cake was collected and dried 
in a vacuum oven at $40{ }^{\circ} \mathrm{C}$ for $24 \mathrm{~h}$ to obtain $0.76 \mathrm{~g}$ dark green product, Bi(p)-(Ani) $)_{4}-\mathrm{COOH}$. Yield: 65.2\%. ${ }^{1} \mathrm{H}-\mathrm{NMR}$ (400 MHz, DMSO-d 6 ), $\delta$, ppm: 10.20 (s, 2H, -CO-NH-), 9.73 (s, 2H, -CO-NH-), 8.09 (s, 4H, Ar-H), 7.62 (m, 8H, Ar-H), 7.43 (s, 6H, -NH-), 6.99 (m, 24H, Ar-H).

Bi(m)-(Ani) $4-\mathbf{C O O H}$ : The synthetic process of $\mathrm{Bi}(\mathrm{m})-(\mathrm{Ani})_{4}-\mathrm{COOH}$ was almost the same as that of $\mathrm{Bi}(\mathrm{p})-(\mathrm{Ani})_{4}-\mathrm{COOH}$. The differences were that terephthalic acid was replaced by isophthalic acid, and isophthalic chloride was obtained and reacted with $\mathrm{LEB} \mathrm{NH}_{2}-(\mathrm{Ani})_{4}-\mathrm{COOH}$ rather than terephthaloyl chloride. Finally, $0.70 \mathrm{~g}$ of $\mathrm{Bi}(\mathrm{m})-(\mathrm{Ani}){ }_{4}-\mathrm{COOH}$ was gained. Yield: $60.1 \% .{ }^{1} \mathrm{H}-\mathrm{NMR}$ (400 MHz, DMSO-d6), , ppm: 10.23 (d, 2H, -CO-NH-), 9.72 (s, 2H, -CO-NH-), 8.51 (s, 1H, Ar-H), 8.13 (d, 2H, Ar-H), 7.79 (s, 1H, Ar-H), 7.69-7.59 (m, 8H, Ar-H), 7.39 (d, 6H, -NH-), 6.96 (m, 24H, Ar-H).

Tri-(Ani)4-COOH: Tri-(Ani)4-COOH can also be obtained by referring to the synthesis process of $\mathrm{Bi}(\mathrm{p})$-(Ani) ${ }_{4}-\mathrm{COOH}$. 1,3,5-benzenetricarboxylic acid was used to get trimesoyl chloride. Then 1.0 mmol trimesoyl chloride was reacted with $3.0 \mathrm{mmol} \mathrm{LEB} \mathrm{NH} 2-(\mathrm{Ani})_{4}-\mathrm{COOH}$. The crude product was dialyzed (MWC 1500) by DMF for two weeks after washed with deionized water, acetone and isopropanol. Yield: 0.90 g, 56.3\%. ${ }^{1} \mathrm{H}-\mathrm{NMR}$ (400 MHz, DMSO-d6), $\delta$, ppm: 10.36 (s, 3H, -CO-NH-), 9.71 (s, 3H, -CO-NH-), 8.64 (s, 3H, Ar-H), 7.96 -7.61 (m, 12H, Ar-H), 7.39 (d, 9H, -NH-), 6.95 (m, $36 \mathrm{H}, \mathrm{Ar}-\mathrm{H})$.

The FTIR spectra of $\mathrm{NH}_{2}-(\mathrm{Ani})_{4}-\mathrm{COOH}, \mathrm{Bi}(\mathrm{p})-(\mathrm{Ani})_{4}-\mathrm{COOH}, \mathrm{Bi}(\mathrm{m})-(\mathrm{Ani})_{4}-\mathrm{COOH}$, and Tri(Ani) ${ }_{4}-\mathrm{COOH}$ are shown in Figure S1. The two characteristic peaks around $3400 \mathrm{~cm}^{-1}$ of NH$_{2}-(\text { Ani) })_{4}$ $\mathrm{COOH}$ belong to the stretching vibrations of $-\mathrm{NH}_{2}$, which is different from $\mathrm{Bi}(\mathrm{p})-(\mathrm{Ani})_{4}-\mathrm{COOH}$, Bi(m)-(Ani) ${ }_{4}-\mathrm{COOH}$, and Tri-(Ani) ${ }_{4}-\mathrm{COOH}$. The peaks located at $1706 \mathrm{~cm}^{-1}$ and $1654 \mathrm{~cm}^{-1} \mathrm{can}^{\mathrm{be}}$ 
assigned to $\mathrm{C}=\mathrm{O}$ stretching vibrations of carboxyl and amide bond, respectively. While $1605 \mathrm{~cm}^{-1}$ and $1504 \mathrm{~cm}^{-1}$ are the stretching vibrations of benzenoid ring skeleton. The peak at $1303 \mathrm{~cm}^{-1} \mathrm{can}$ be assigned to stretching vibrations of $\mathrm{C}-\mathrm{N}$ bond linked with benzenoid ring. The relatively stronger peak between $700 \mathrm{~cm}^{-1}$ and $900 \mathrm{~cm}^{-1}$, located at $820 \mathrm{~cm}^{-1}$, indicates that the benzenoid ring is substituted in the para position. There are other peaks around $700 \sim 900 \mathrm{~cm}^{-1}$ of Bi(m)-(Ani) ${ }_{4}-\mathrm{COOH}$ and Tri-(Ani) ${ }_{4}-\mathrm{COOH}$, which are consist with the characteristics of intermediate meta-substituted and tri-substituted benzenes.

The MALDI-TOF MS of $\mathrm{NH}_{2}-(\mathrm{Ani})_{4}-\mathrm{COOH}, \mathrm{Bi}(\mathrm{p})-(\mathrm{Ani})_{4}-\mathrm{COOH}, \mathrm{Bi}(\mathrm{m})-(\mathrm{Ani})_{4}-\mathrm{COOH}$ and Tri-(Ani)4-COOH are 480.5, 1092.6, 1092.7 and 1599.9 (Figure S2(a)-Figure S5(a)), which are consist well with ideal values of 481.2, 1092.4, 1092.4, 1599.6. 


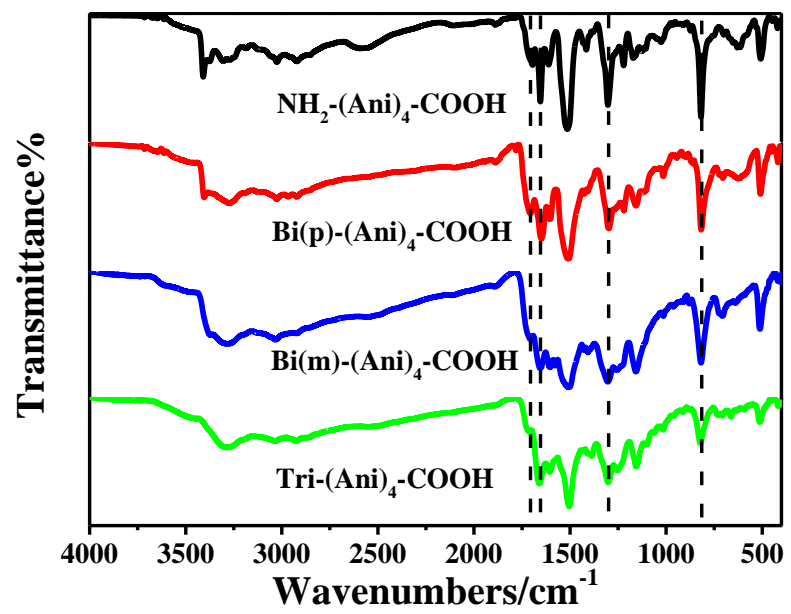

Figure S1. FTIR spectra of LEB (a) $\mathrm{NH}_{2}-(\mathrm{Ani})_{4}-\mathrm{COOH}$; (b) Bi(p)-(Ani) $)_{4}-\mathrm{COOH}$; (c) Bi(m)-(Ani) $)_{4}-\mathrm{COOH}$; and (d) Tri-(Ani) $4-\mathrm{COOH}$. 


\section{1. $\mathrm{LEB} \mathrm{NH}$-(Ani) 4 -COOH}
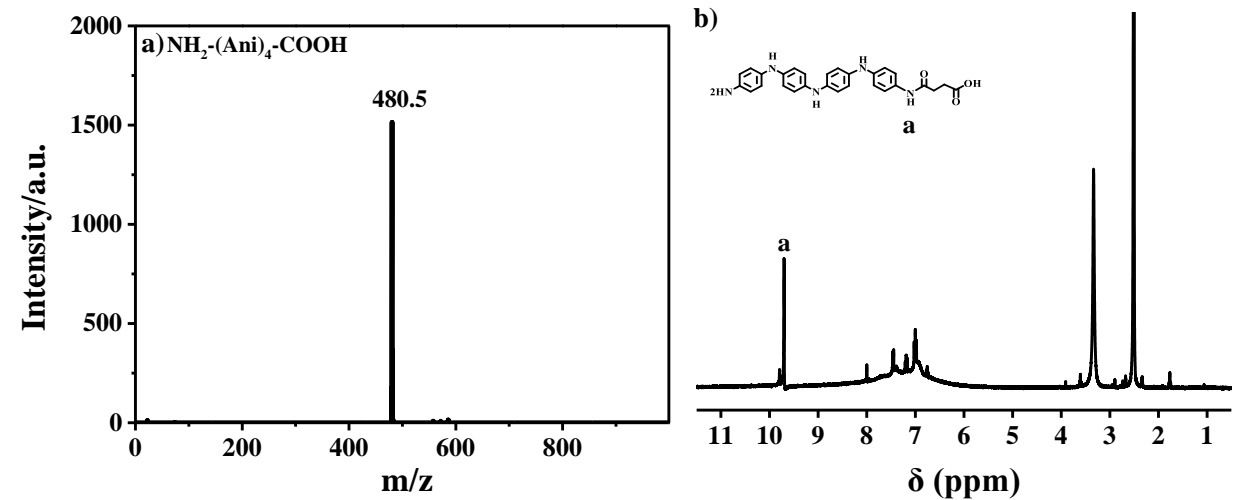

Figure S2. (a) MALDI MS spectrum and (b) ${ }^{1} \mathrm{H}$ NMR spectrum of LEB $\mathrm{NH}_{2}-(\mathrm{Ani})_{4}-\mathrm{COOH}$.

\section{LEB Bi(p)-(Ani) $)_{4}-\mathrm{COOH}$}
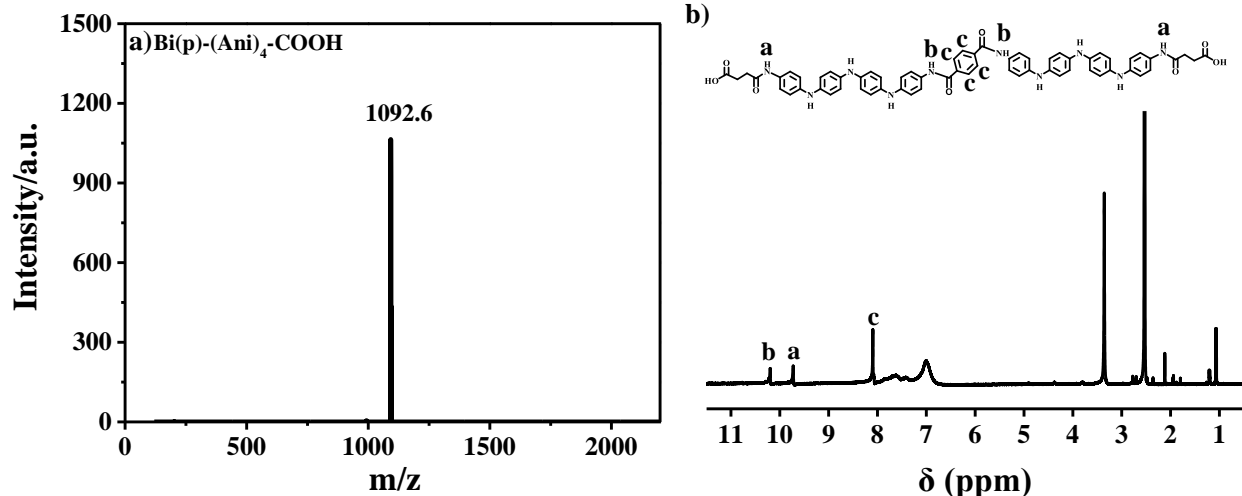

Figure S3. (a) MALDI MS spectrum and (b) ${ }^{1} \mathrm{H}$ NMR spectrum of LEB Bi(p)-(Ani) ${ }_{4}-\mathrm{COOH}$. 


\section{LEB Bi(m)-(Ani) $4-\mathrm{COOH}$}
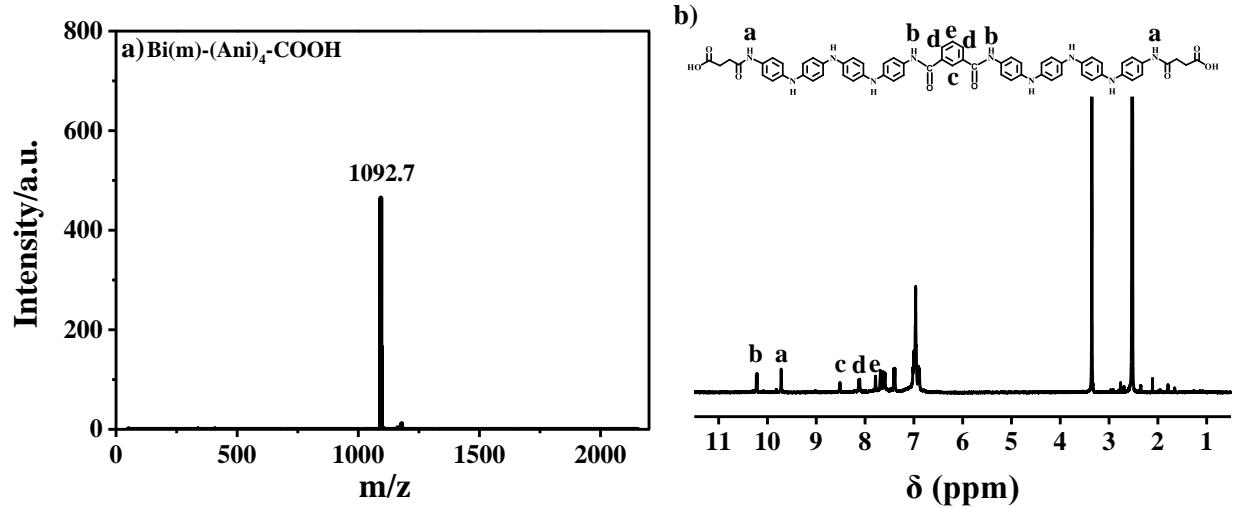

Figure S4. (a) MALDI MS spectrum and (b) ${ }^{1} \mathrm{H}$ NMR spectrum of LEB Bi(m)-(Ani) ${ }_{4}-\mathrm{COOH}$.

\section{LEB Tri-(Ani) $4-\mathrm{COOH}$}
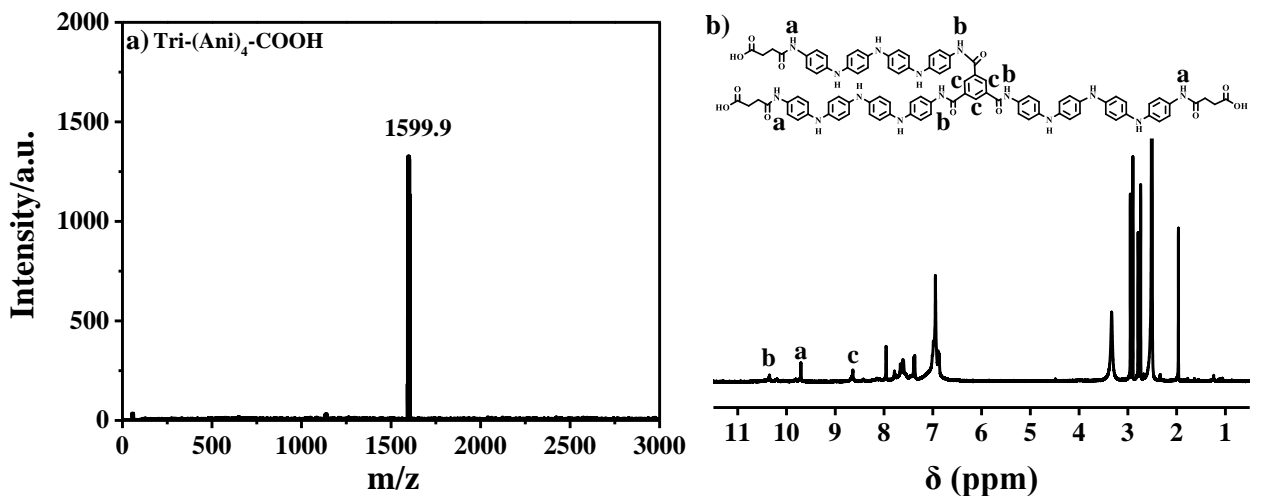

Figure S5. (a) MALDI MS spectrum and (b) ${ }^{1} \mathrm{H}$ NMR spectrum of LEB Tri-(Ani) $4-\mathrm{COOH}$. 

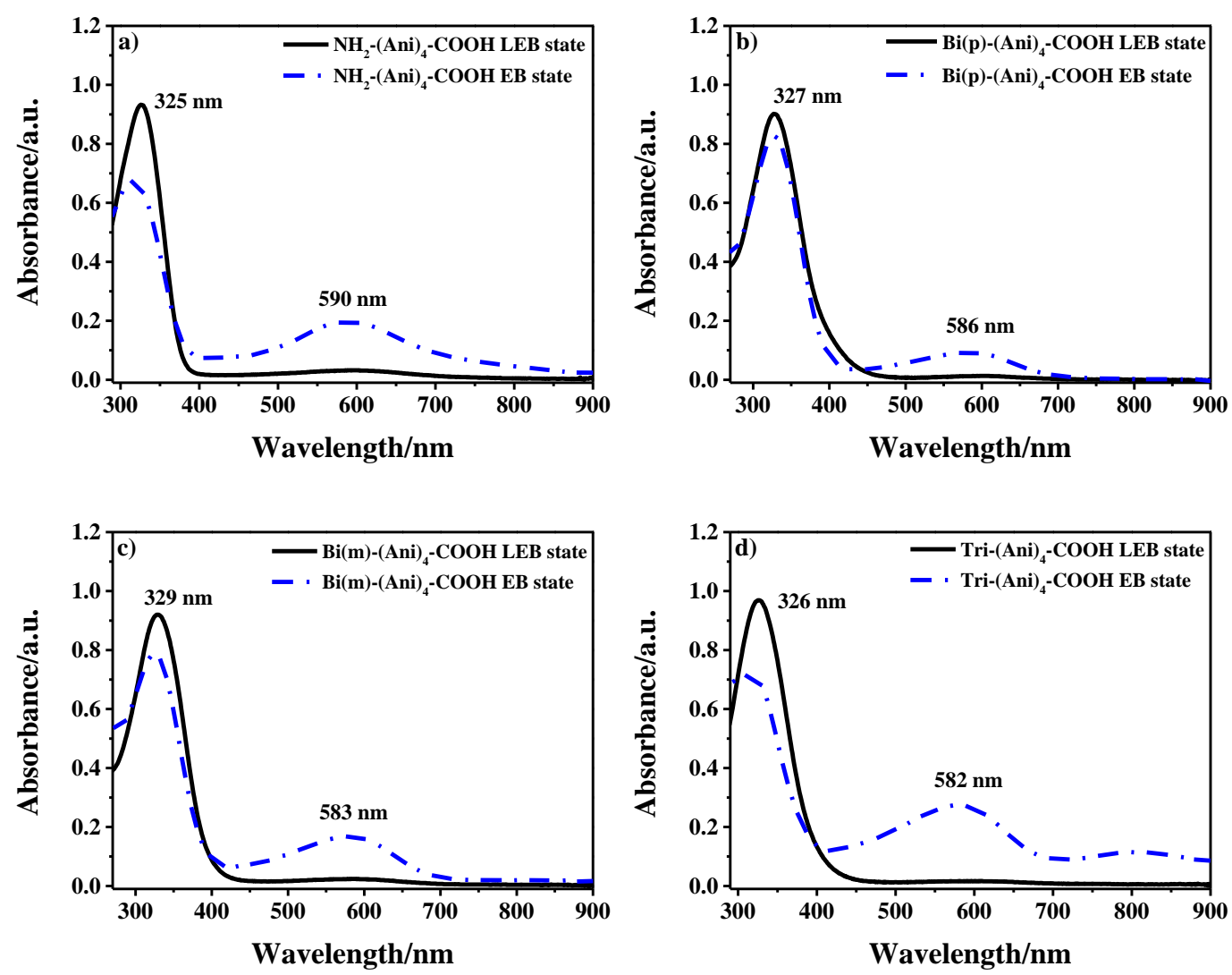

Figure S6. UV-vis absorbance spectra of (a) $\mathrm{NH}_{2}-(\mathrm{Ani})_{4}-\mathrm{COOH}$; (b) Bi(p)-(Ani) ${ }_{4}-\mathrm{COOH}$; (c) Bi(m)-(Ani) 4- $^{-}$ $\mathrm{COOH}$; and (d) Tri-(Ani) $)_{4}-\mathrm{COOH}$ in DMF solution in LEB and EB state, respectively. 


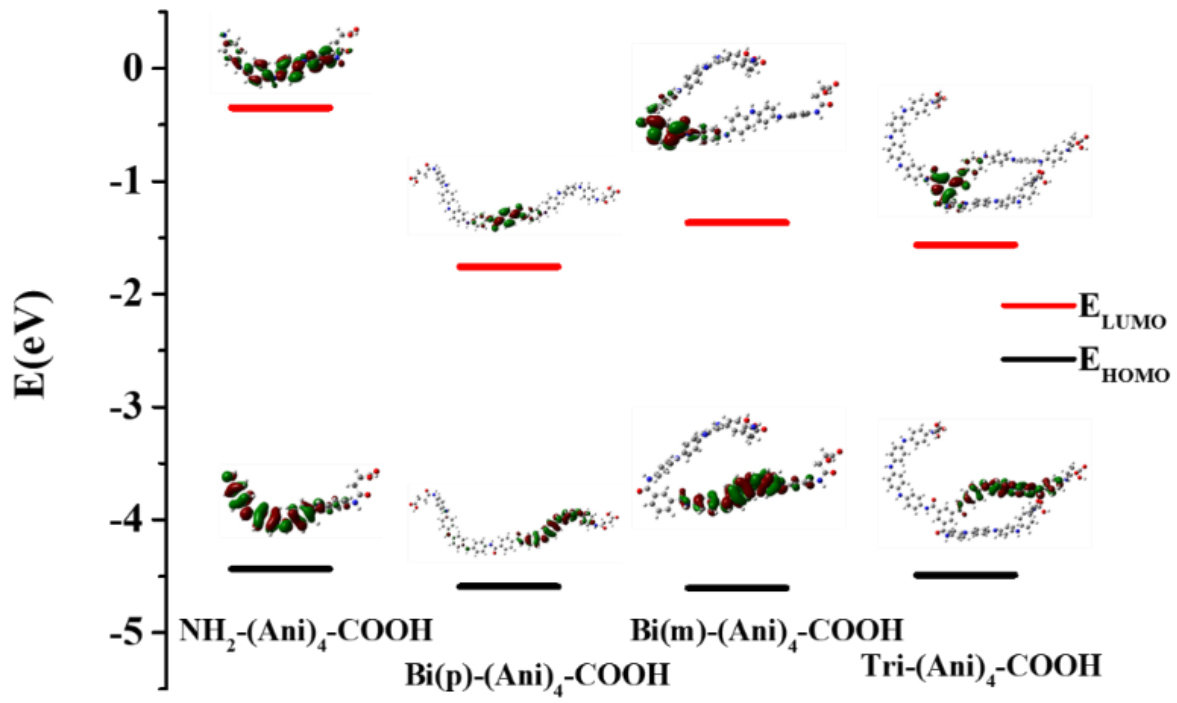

Figure S7. Energy levels and electron cloud distribution of the four tetraaniline derivatives. 

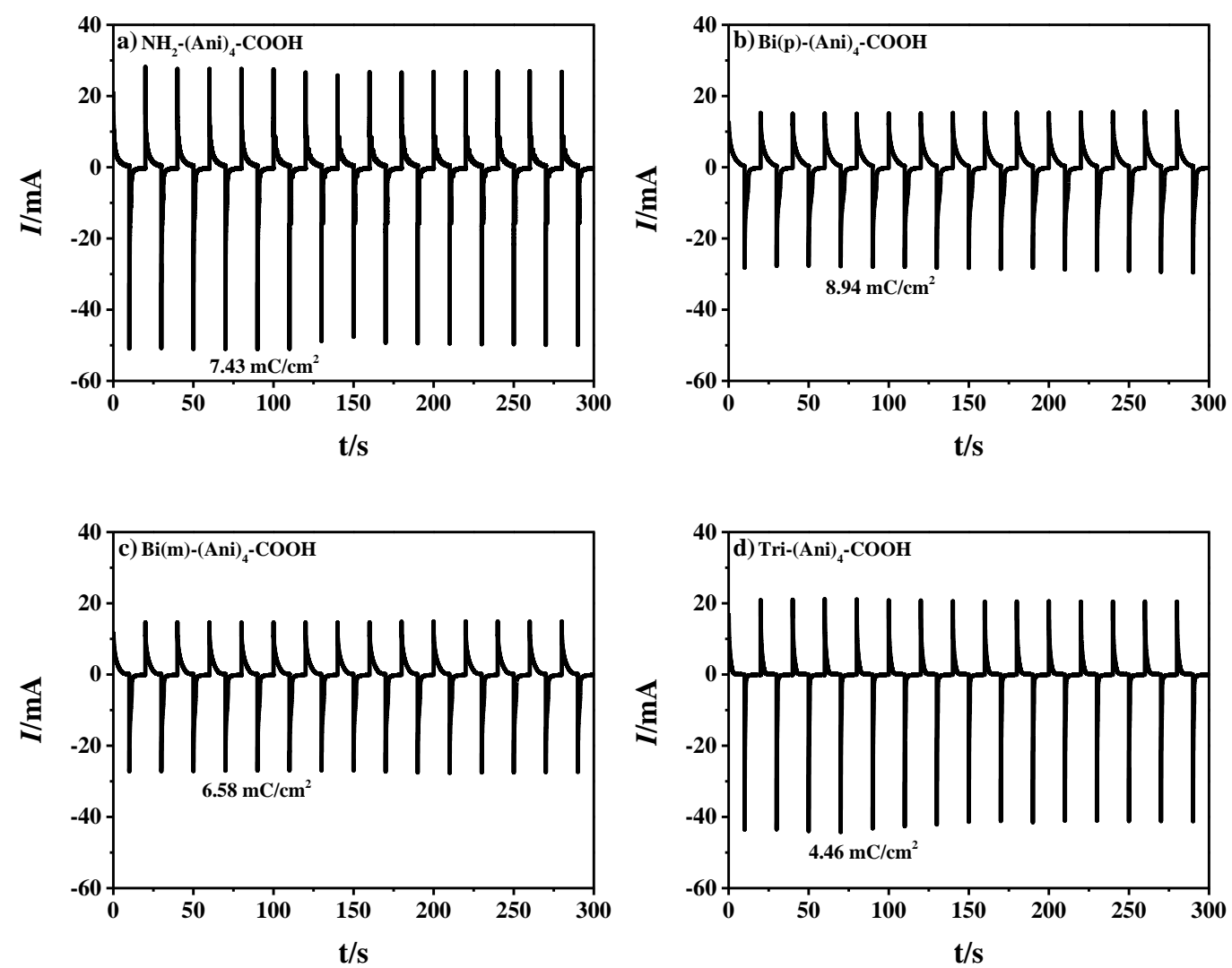

Figure S8. I-t curves of (a) $\mathrm{NH}_{2}-(\mathrm{Ani})_{4}-\mathrm{COOH}$; (b) Bi(p)-(Ani) ${ }_{4}-\mathrm{COOH}$; (c) $\mathrm{Bi}(\mathrm{m})-(\mathrm{Ani})_{4}-\mathrm{COOH}$; and (d) Tri(Ani) $)_{4}-\mathrm{COOH}$ films in $1.0 \mathrm{~mol} / \mathrm{L} \mathrm{HCl}$ solution between -0.2 and $+0.6 \mathrm{~V}$. 

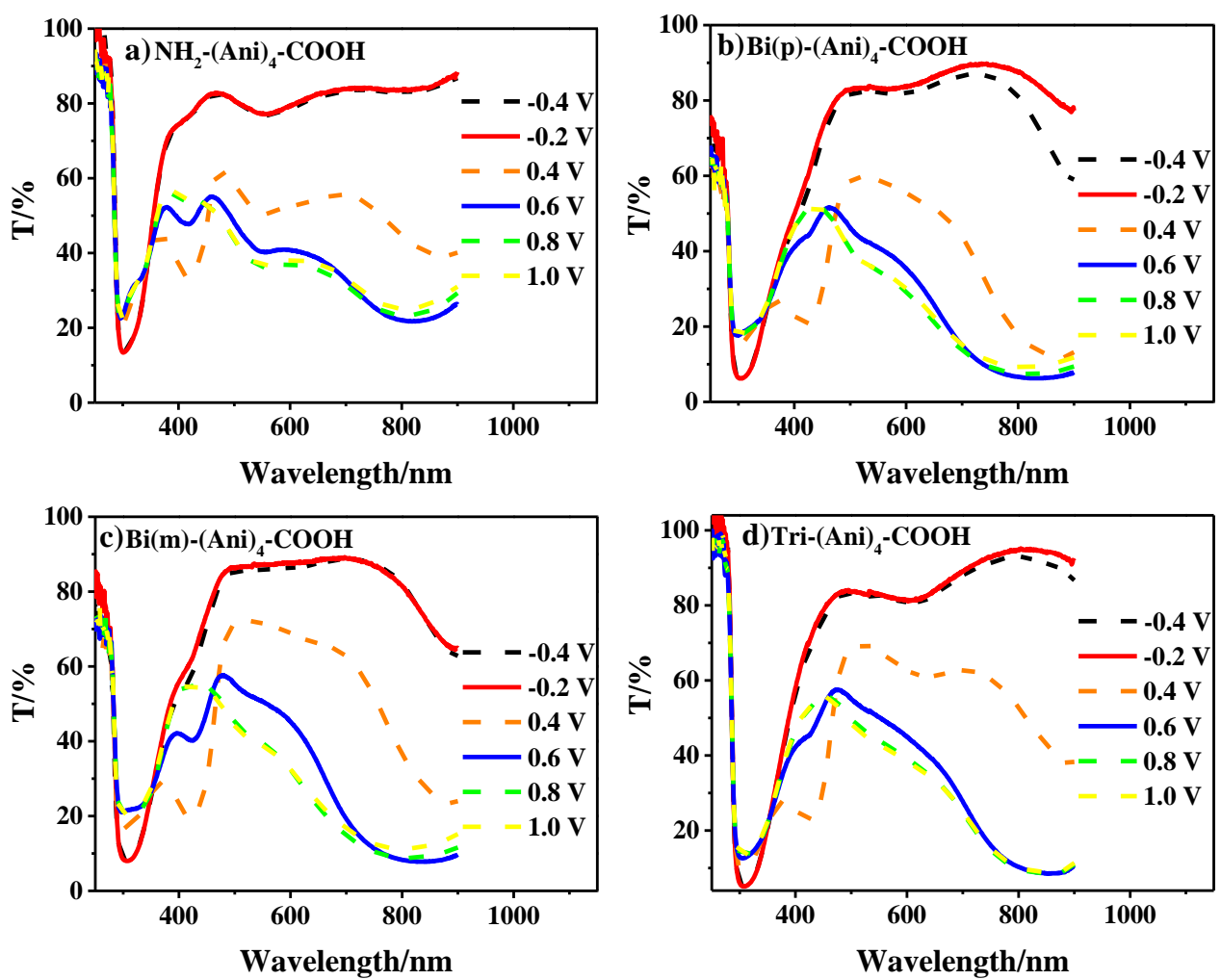

Figure S9. Transmittance spectra of (a) $\mathrm{NH}_{2}-(\mathrm{Ani})_{4}-\mathrm{COOH}$; (b) $\mathrm{Bi}(\mathrm{p})-(\mathrm{Ani})_{4}-\mathrm{COOH}$; (c) $\mathrm{Bi}(\mathrm{m})-(\mathrm{Ani})_{4}-\mathrm{COOH}$; and (d) Tri-(Ani) $)_{4}-\mathrm{COOH}$ films at different potentials in $1 \mathrm{~mol} / \mathrm{L} \mathrm{HCl}$ solution. 
Table S1. CIE results for the four tetraaniline derivative films.

\begin{tabular}{ccc}
\hline Product/ Potential$(\mathrm{V})$ & $\mathrm{x}$ & $\mathrm{y}$ \\
\hline $\mathrm{NH}_{2}-(\mathrm{Ani})_{4}-\mathrm{COOH} /-0.4$ & 0.3323 & 0.3293 \\
$\mathrm{NH}_{2}-(\mathrm{Ani})_{4}-\mathrm{COOH} /-0.2$ & 0.3327 & 0.3295 \\
$\mathrm{NH}_{2}-(\mathrm{Ani})_{4}-\mathrm{COOH} / 0.4$ & 0.3366 & 0.3447 \\
$\mathrm{NH}_{2}-(\mathrm{Ani})_{4}-\mathrm{COOH} / 0.6$ & 0.3103 & 0.3076 \\
$\mathrm{NH}_{2}-(\mathrm{Ani})_{4}-\mathrm{COOH} / 0.8$ & 0.3046 & 0.2948 \\
$\mathrm{NH}_{2}-(\mathrm{Ani})_{4}-\mathrm{COOH} / 1.0$ & 0.3077 & 0.2963 \\
$\mathrm{Bi}(\mathrm{p})-(\mathrm{Ani})_{4}-\mathrm{COOH} /-0.4$ & 0.3456 & 0.3544 \\
$\mathrm{Bi}(\mathrm{p})-(\mathrm{Ani})_{4}-\mathrm{COOH} /-0.2$ & 0.3445 & 0.3511 \\
$\mathrm{Bi}(\mathrm{p})-(\mathrm{Ani})_{4}-\mathrm{COOH} / 0.4$ & 0.3590 & 0.3971 \\
$\mathrm{Bi}(\mathrm{p})-(\mathrm{Ani})_{4}-\mathrm{COOH} / 0.6$ & 0.2976 & 0.3153 \\
$\mathrm{Bi}(\mathrm{p})-(\mathrm{Ani})_{4}-\mathrm{COOH} / 0.8$ & 0.2828 & 0.2909 \\
$\mathrm{Bi}(\mathrm{p})-(\mathrm{Ani})_{4}-\mathrm{COOH} / 1.0$ & 0.2863 & 0.2923 \\
$\mathrm{Bi}(\mathrm{m})-(\mathrm{Ani})_{4}-\mathrm{COOH} /-0.4$ & 0.3467 & 0.3555 \\
$\mathrm{Bi}(\mathrm{m})-(\mathrm{Ani})_{4}-\mathrm{COOH} /-0.2$ & 0.3450 & 0.3526 \\
$\mathrm{Bi}(\mathrm{m})-(\mathrm{Ani})_{4}-\mathrm{COOH} / 0.4$ & 0.3678 & 0.4066 \\
$\mathrm{Bi}(\mathrm{m})-(\mathrm{Ani})_{4}-\mathrm{COOH} / 0.6$ & 0.3152 & 0.3418 \\
$\mathrm{Bi}(\mathrm{m})-(\mathrm{Ani})_{4}-\mathrm{COOH} / 0.8$ & 0.2840 & 0.2973 \\
$\mathrm{Bi}(\mathrm{m})-(\mathrm{Ani})_{4}-\mathrm{COOH} / 1.0$ & 0.2868 & 0.2973 \\
Tri-(Ani ${ }_{4}-\mathrm{COOH} /-0.4$ & 0.3372 & 0.3439 \\
Tri-(Ani $)_{4}-\mathrm{COOH} /-0.2$ & 0.3362 & 0.3412 \\
Tri-(Ani) ${ }_{4}-\mathrm{COOH} / 0.4$ & 0.3604 & 0.4006 \\
Tri-(Ani $)_{4}-\mathrm{COOH} / 0.6$ & 0.3132 & 0.3317 \\
Tri-(Ani $)_{4}-\mathrm{COOH} / 0.8$ & 0.3010 & 0.3106 \\
Tri-(Ani) $)_{4}-\mathrm{COOH} / 1.0$ & 0.3008 & 0.3080 \\
\hline
\end{tabular}




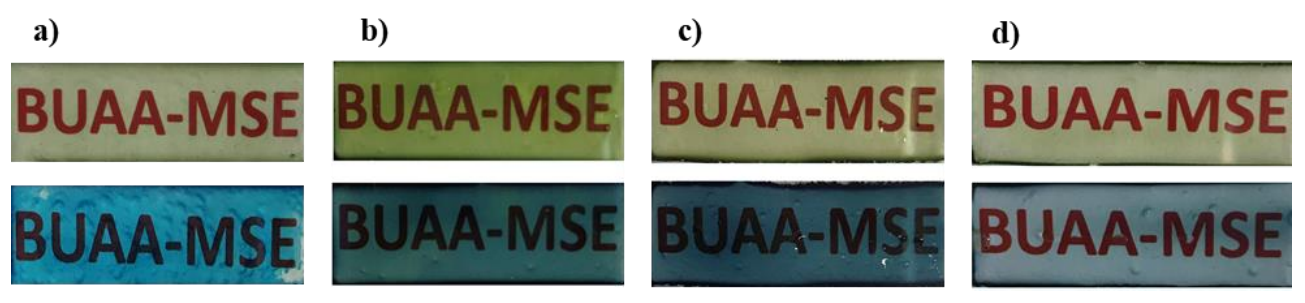

Figure S10. Photos of (a) $\mathrm{NH}_{2}-(\mathrm{Ani})_{4}-\mathrm{COOH}$; (b) Bi(p)-(Ani) $)_{4}-\mathrm{COOH}$; (c) Bi(m)-(Ani) 4 -COOH; and (d) Tri(Ani) $)_{4}-\mathrm{COOH}$ films at -0.2 and $+0.6 \mathrm{~V}$.

Table S2. EIS results for the four tetraaniline derivative films.

\begin{tabular}{ccccccc}
\hline Product & $\mathrm{R} 1(\Omega)$ & $\mathrm{C} 1-\mathrm{T}\left(\times 10^{-3} \mathrm{~F}\right)$ & $\mathrm{C} 1-\mathrm{P}(\mathrm{F})$ & $\mathrm{R} 2(\Omega)$ & $\mathrm{C} 2-\mathrm{T}\left(\times 10^{-3} \mathrm{~F}\right)$ & $\mathrm{C} 2-\mathrm{P}(\mathrm{F})$ \\
\hline $\mathrm{NH}_{2}-(\mathrm{Ani})_{4}-\mathrm{COOH}$ & 21.57 & 0.71 & 0.65 & 173.40 & 3.59 & 0.82 \\
$\mathrm{Bi}(\mathrm{p})-\left(\right.$ Ani) ${ }_{4}-\mathrm{COOH}$ & 15.75 & 0.08 & 0.91 & 50.39 & 4.60 & 0.72 \\
$\mathrm{Bi}(\mathrm{m})-(\mathrm{Ani})_{4}-\mathrm{COOH}$ & 11.91 & 0.32 & 0.67 & 37.74 & 8.72 & 0.77 \\
Tri-(Ani) $)_{4}-\mathrm{COOH}$ & 13.99 & 0.20 & 0.72 & 33.68 & 5.68 & 0.69 \\
\hline
\end{tabular}



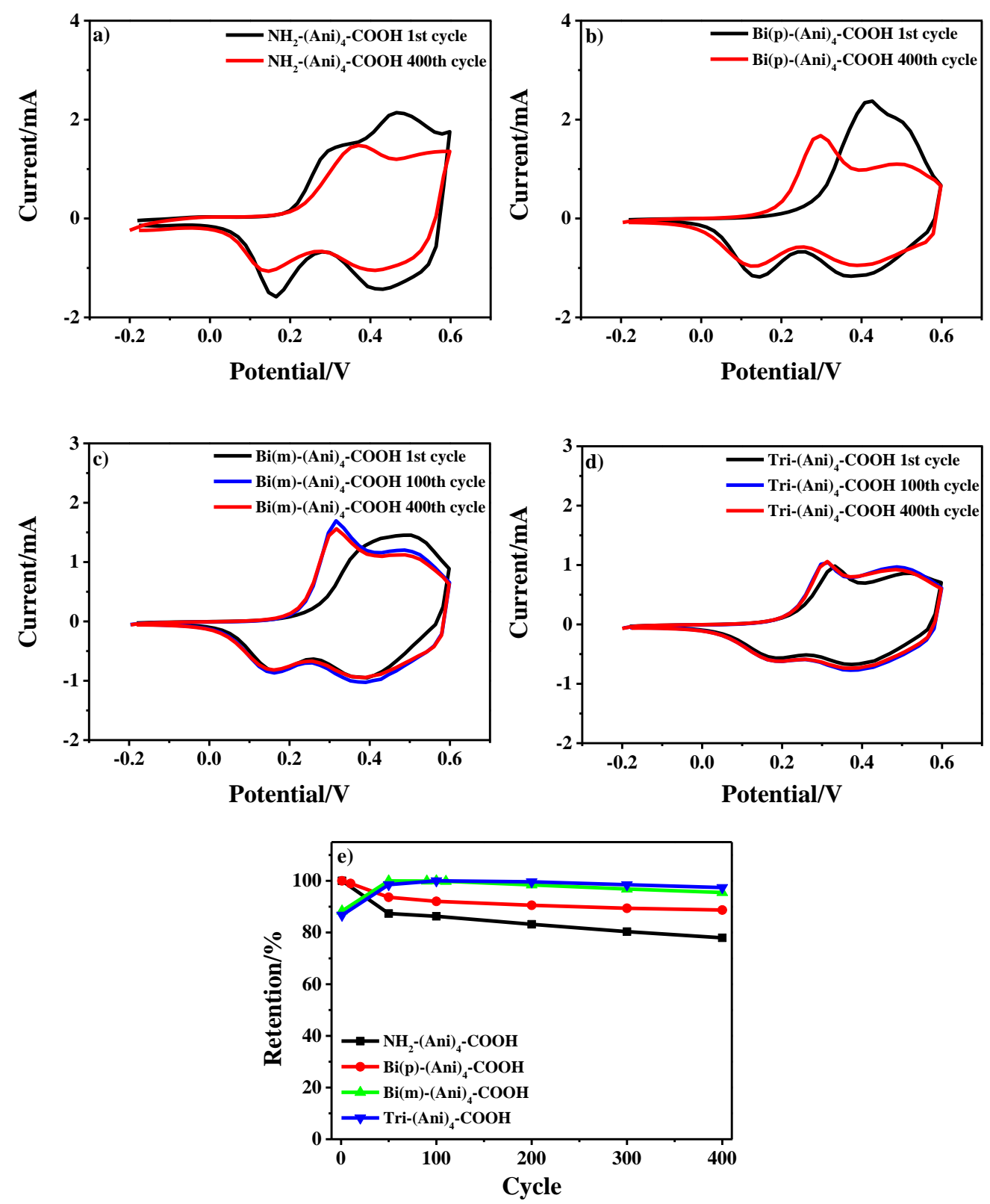

Figure S11. CV curves of (a) $\mathrm{NH}_{2}-(\mathrm{Ani})_{4}-\mathrm{COOH}$; (b) Bi(p)-(Ani) $)_{4}-\mathrm{COOH}$; (c) Bi(m)-(Ani) ${ }_{4}-\mathrm{COOH}$; and (d) Tri(Ani) ${ }_{4}-\mathrm{COOH}$ films in $1.0 \mathrm{~mol} / \mathrm{L}$ flowing $\mathrm{HCl}$ solution at scan rate of $100 \mathrm{mV} / \mathrm{s}$. e) The charge retention curves of them during $\mathrm{CV}$ cycles. 

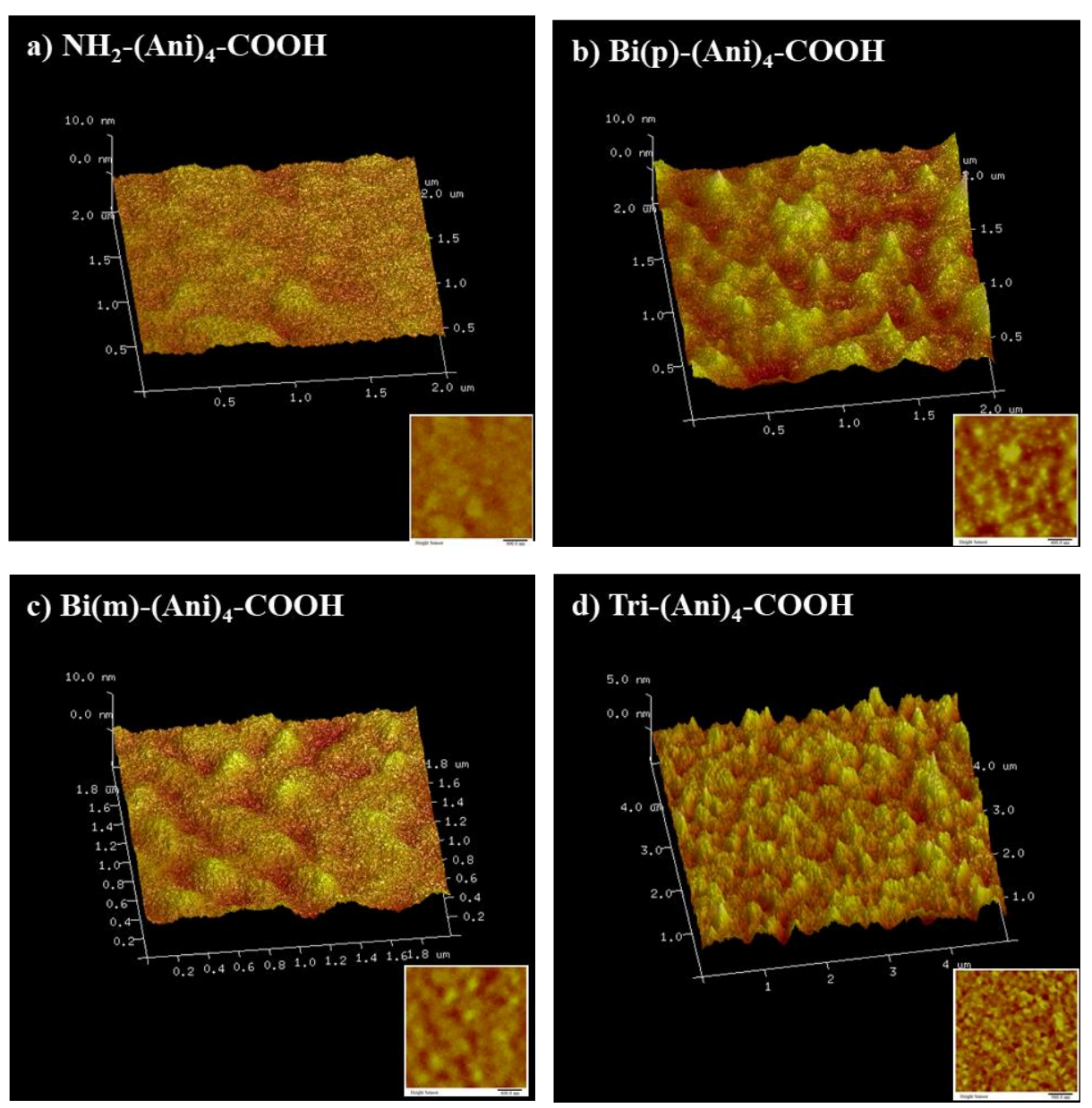

Figure S12. AFM images of (a) $\mathrm{NH}_{2}-(\mathrm{Ani})_{4}-\mathrm{COOH}$; (b) Bi(p)-(Ani) $)_{4}-\mathrm{COOH}$; (c) Bi(m)-(Ani) $)_{4}-\mathrm{COOH}$; and (d) Tri-(Ani) $)_{4}-\mathrm{COOH}$ films before $\mathrm{CV}$ cycle test. Inset: 2D height contrast. 

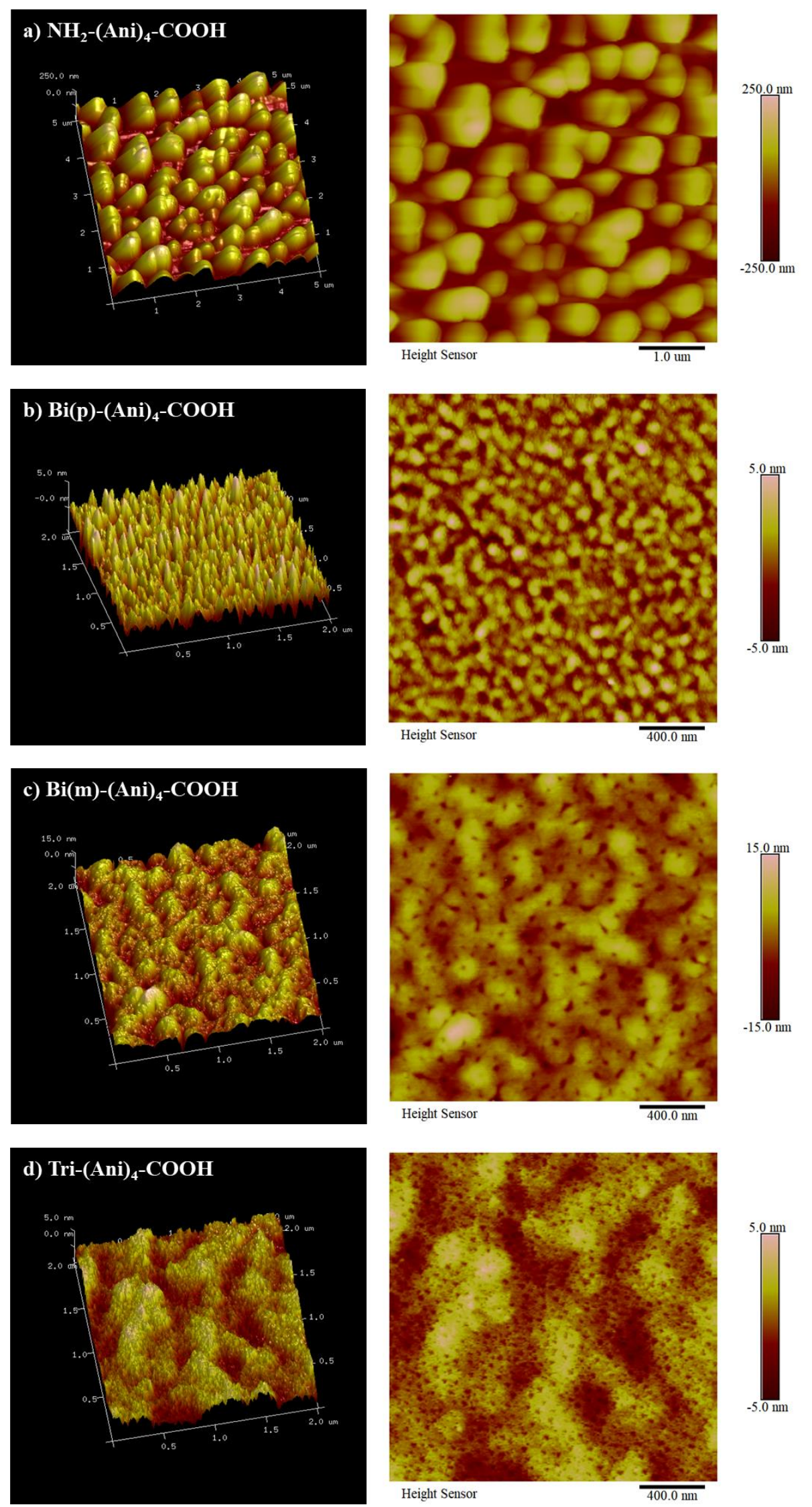

Figure S13. 3D and 2D height contrast AFM images of (a) $\mathrm{NH}_{2}-(\mathrm{Ani})_{4}-\mathrm{COOH}$; (b) Bi(p)-(Ani) $)_{4}-\mathrm{COOH}$; (c) $\mathrm{Bi}(\mathrm{m})-(\mathrm{Ani})_{4}-\mathrm{COOH}$; and (d) Tri-(Ani) ${ }_{4}-\mathrm{COOH}$ films on FTO in $0.5 \mathrm{~mol} / \mathrm{L} \mathrm{H}_{2} \mathrm{SO}_{4}$ solution after $3000 \mathrm{CV}$ cycles. 

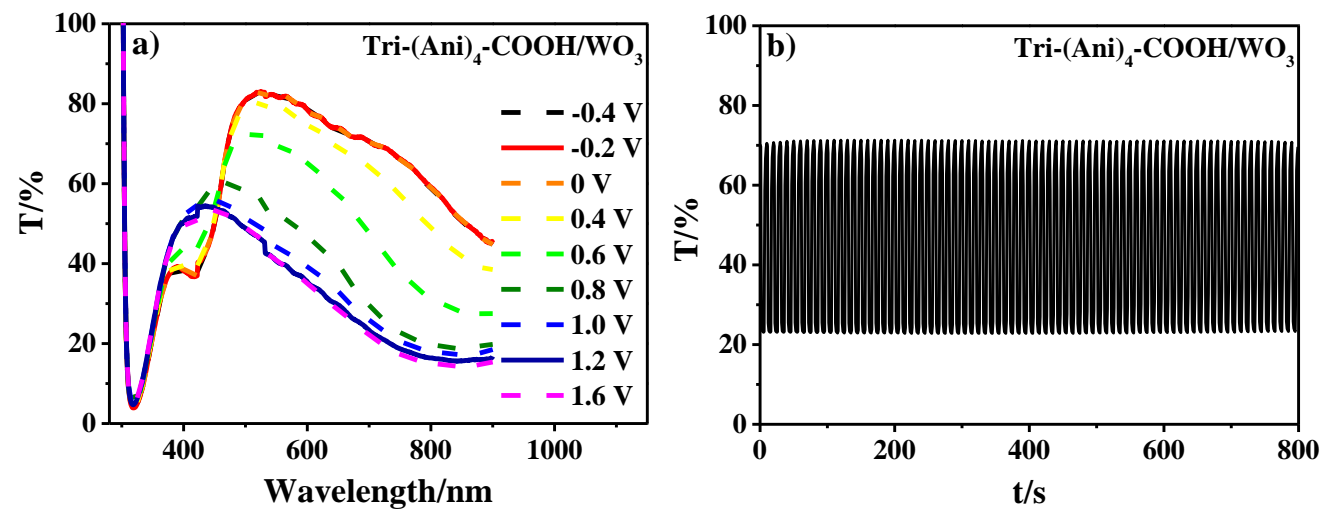

c)

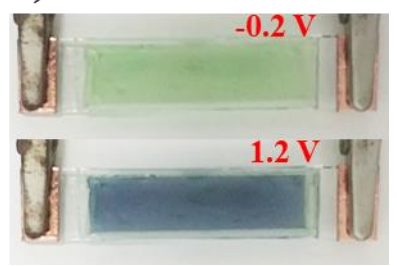

Figure S14. (a) Transmittance spectra, (b) switching response curve and (c) photos of the Tri-(Ani) $)_{4}-\mathrm{COOH} / \mathrm{WO}_{3}$ ECD.

\section{REFERENCES}

(1) Cao, L.; Gong, C.; Yang, J. Conducting tetraaniline derivatives with fast switching time, enhanced contrast and coloration efficiency. Electrochim. Acta 2016, 192, 422-430.

(2) Wang, M.; Wang, K.; Liu, B.; Lu, H.; Huang, M.; Yang, J. Effect of terminal groups on the microscopic morphologies and electrochromic properties of pentaaniline derivatives. Synth. Met. 2019, 251, 15-23. 